\title{
Partial widths and interchannel coupling in autoionizing states in terms of complex eigenvalues and complex coordinates
}

\author{
Cleanthes A. Nicolaides and Theodoros Mercouris \\ Theoretical and Physical Chemistry Institute, National Hellenic Research Foundation, \\ 48, Vassileos Constantinou Avenue, Athens, 116/35, Greece
}

(Received 1 March 1985)

\begin{abstract}
The use of complex coordinates allows the possibility of treating resonances of many-electron systems based on a complex eigenvalue Schrödinger equation. A many-body analysis of this equation has led to the establishment of convenient and systematic procedures for including electron correlation and for computing partial and total widths, without and with interchannel coupling, in multichannel autoionization. This is done by computing the asymptotic-pair (AP) correlation functions from an independent asymptotic-pair approximation (IAPA) and then mixing them (MAP, i.e., the mixing of asymptotic pairs) from a diagonalization of the total non-Hermitian Hamiltonian matrix. Nonorthonormality complications are resolved by straightforward computation. We report results for $\mathrm{Ne}^{+} 1 s 2 s^{2} 2 p^{62} S$, which decays into five one-electron channels: $1 s-2 p^{2}{ }^{1} D, 1 s-2 p^{2}{ }^{1} S, 1 s-$ $2 s 2 p^{3} P^{o}, 1 s-2 s 2 p^{1} P^{o}$, and $1 s-2 s^{2}{ }^{1} S$. The corresponding partial-width values from MAP are (in $10^{-2}$ a.u.): $0.565,0.040,0.032,0.165$, and 0.043 . The proximity of these results to the available experimental information as well as that to previous results from many-body calculations with real coordinates offers a practical verification of this theory.
\end{abstract}

\section{INTRODUCTION}

The phenomenon of autoionization and the corresponding existence of quasilocalized states in the continuous spectrum of many-electron systems have intriguing mathematical and physical properties. This is due mainly to their forming part of the continuous spectrum and to their possible strong deviations from the independentparticle model description. An excellent recent review of aspects of this field has been published by Aberg and Howat. ${ }^{1}$

In a series of papers, ${ }^{2-15}$ we have published our findings as regards the formalism, certain properties, the electronic structure, and the practical computation of resonances and autoionizing states. Our emphasis has been on general approaches suitable for multichannel resonances of arbitrary structure and number of electrons.

In this paper we continue our investigations of autoionizing states as eigenfunctions of the nonrelativistic nonHermitian Hamiltonian with complex coordinates and complex eigenvalues. The problem we have tackled involves the multichannel decay of an inner hole state $\left(\mathrm{Ne}^{+}\right.$ $1 s 2 s^{2} 2 p^{62} S$ ) with the object of computing its partial and total widths, without and with interchannel coupling.

The emergence of the use of complex coordinates either in the Hamiltonian or in the wave function, has presented, in principle, an elegant alternative to the calculation of resonant phenomena, via the application of square integrable function spaces only. ${ }^{5,13,16-23}$ However, even though the theory is available_-just as the $N$-particle Schrödinger equation is available-its coarse implementation (i.e., repeated diagonalization of large matrices and search for root stabilization) to real, many-electron systems is practically impossible. For example, the recent review by $\mathrm{Ho}^{16}$ contains numerical results for single-channel, two- or three-electron systems only. There, he, like many other researchers in this field, emphasized the need for going through the bottleneck of many-electron partial-width computation for real systems. Some theoretical results have been produced, but only for model systems. ${ }^{17,18}$

The approach introduced in Refs. 5 and 7 allows the practical analysis and calculation of partial widths in real, multichannel systems. In essence, this approach has introduced into the field of the complex eigenvalue treatment of resonances notions of many-body theory applied before for bound states as well as proposals for separation and choice and optimization of function spaces in the complex energy-coordinate planes. The first numerical application was published recently on the $\mathrm{Ne}^{+}$ $1 s 2 s^{2} 2 p^{62} S$ state. ${ }^{13}$ The work presented here incorporates it and extends it.

\section{REVIEW OF THEORY}

The many-electron theory of resonances in terms of complex coordinates which we have used has been presented in detail before. ${ }^{5,7,13,14}$ Here, we emphasize some of its basic ideas and results which are relevant to this application. Reference to other methods and to the difficulties as regards the many-electron problem with complex eigenvalues can be found in Refs. 5, 7, 13, 14, and 16.

The aim is to solve the many-electron Schrödinger equation with a complex eigenvalue $z$ and an unnormalizable resonance function $\Psi$ :

$$
\begin{aligned}
& H \Psi=z \Psi, \\
& z=E-(i / 2) \Gamma .
\end{aligned}
$$


$E$ is the total energy and $\Gamma$ is the total width due to interactions in $H$. For the decay of one particle of coordinate $r, \Psi$ has the following exact asymptotic form: ${ }^{19,7}$

$$
\Psi\left(r ; K_{0}\right) \underset{r \rightarrow \infty}{\sim}-V a\left[\frac{2 \pi}{K_{0}}\right]^{1 / 2} \exp \left[i\left(K_{0} r+\delta\right)\right],
$$

where $V$ is inversely proportional to the lifetime, $a^{2}$ is the weight of the localized component [see Eq. (5)], $K_{0}$ is the complex momentum of the emitted particle and $\delta$ is the phase shift.

Continuation into the complex coordinate plane, $\rho=r e^{i \vartheta}$, results in normalizable wave functions and regularized matrix elements ${ }^{20}$ and a new equation with a nonHermitian Hamiltonian (e.g., Refs. 20-22, 4, 5, 7):

$$
H(\rho) \Psi(\rho)=z \Psi(\rho) .
$$

The square integrable $\Psi(\rho)$ contains components which contribute to the stability of the state and components which contribute to its decay. A physically and computationally motivated theory must differentiate between the function spaces describing the two types and their optimization procedures (see also Ref. 23). According to Refs. 5 and $7, \Psi(\rho)$ can correspondingly be separated into two parts, the localized $\Psi_{0}$ and the asymptotic $X$ parts,

$$
\Psi(\rho)=a \Psi_{0}(\rho)+b X(\rho)
$$

with the following $[(\mathrm{a})-(\mathrm{g})]$ properties.

(a) We have

$$
\left\langle\Psi_{0}(\rho)|H(\rho)| \Psi_{0}(\rho)\right\rangle=\left\langle\Psi_{0}(r)|H(r)| \Psi_{0}(r)\right\rangle=E_{0},
$$

where $E_{0}$ is real and $\Psi_{0}(r)$ is the exact square integrable $N$-electron wave function describing the initially localized state. 2,5

(b) We have

$$
z=E_{0}+\Delta-(i / 2) \Gamma,
$$

where $\Delta$ is the energy shift and $\Gamma$ is the width due to the mixing of $\Psi_{0}$ with $X$.

(c) On resonance,

$$
\left|\frac{b}{a}\right|^{2}=\frac{\Gamma}{\left|K_{0}\right|} \text {. }
$$

(d) The terms in $\Psi_{0}(\rho)$ contain only hydrogenic or Gaussian orbitals, while the terms in $X(\rho)$ contain Gamow orbitals ${ }^{5,7}$ as well, which enter into the asymptotic correlation functions. $5,7,13,19$

(e) Relations (5)-(7) imply that, in solving for Eq. (4), the calculation of $\Psi_{0}$ can be done only once, on the real axis. ${ }^{5}$ Given the nature of the decay phenomenon, the effect of the continuum is taken to act as a perturbation on $\dot{\Psi}_{0}$. The calculations in the complex plane are thus much smaller and feasible for any system and concern only the optimization of few terms in $X(\rho)$ and the mixing coefficients $a$ and $b .^{24}$

(f) If $\Psi(\rho)$ is expanded in terms of a subshell cluster expansion, each decay channel in a multichannel autoionization process is represented by an asymptotic-pair correlation function $\Pi_{\mathrm{as}}\left(\rho_{1} \rho_{2}\right)$ (for single-electron emission). By introducing into the quantum mechanics of multichannel resonances the independent asymptotic-pair approximation (IAPA), we obtain the partial widths from the calculation of each such asymptotic-pair correlation function. ${ }^{7,13}$

(g) The calculation of $\Psi_{0}$ on the real axis is guided by three basic criteria, introduced in Ref. 2 (see also Ref. 5).

(i) The criterion of an optimized zeroth-order representation based on the notion of localization (see below).

(ii) The criterion of orthogonality to lower states of the same symmetry (see Refs. 2 and 5).

(iii) The criterion of analysis of electron-electron interactions according to their energy and wave-function importance (see, e.g., Refs. 2, 6, 13, and 25).

The first criterion is required for the identification of $\Psi_{0}$ with a resonance. The notion of localization is fundamental to the existence of resonances. The virial theorem and (for atoms) the number of nodes serve as guiding principles. A computational scheme which satisfies both is the Hartree-Fock (HF) or the multiconfigurational HF (MCHF) approaches. Due to their self-consistent character, dynamical screening, and essential correlation effects can be incorporated into the resonance wave functions rigorously, at the zeroth-order level.

Thus, the first aim is the calculation of a state-specific HF or MCHF wave function which satisfies the virial theorem and has converged well (not always easy for negative-ion resonances). Unstable solutions suggest very broad or nonexistent resonances. ${ }^{3,26}$ The remaining localized correlation, which contributes to the stability of the state, is added variationally [e.g., (6)] and convergence to a "stable root" is guaranteed. ${ }^{27-34}$

\section{PARTIAL WIDTHS AND INTERCHANNEL COUPLING FROM THE MIXING OF THE ASYMPTOTIC PAIRS (MAP)}

It has already been demonstrated ${ }^{5,7,13}$ that, for oneelectron emission, the real process of autoionization for each decay channel is described in terms of the symmetry adapted asymptotic-pair correlation function, $\Pi_{\text {as }}\left(\rho_{1} \rho_{2}\right)$. The form of each $\Pi_{\text {as }}$ is found by first analyzing $\Psi_{0}$ in terms of its HF or MCHF zeroth order and the various correlation vectors. Each $\Pi_{\text {as }}$ is expressed in terms of Slater (Gaussian) and Gamow orbitals whose nonlinear parameters are optimized subject to the constraint of Eq. (8) and the virial condition, for each $\Pi_{\text {as }}$ separately.

Within this independent asymptotic-pair approximation (IAPA) for each channel $i$ there corresponds a $\Pi_{\text {as }}^{i}$, a par-

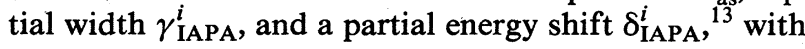

$$
\begin{aligned}
& \Gamma_{\mathrm{IAPA}}=\sum_{i} \gamma_{\mathrm{IAPA}}^{i}, \\
& \Delta_{\mathrm{IAPA}}=\sum_{i} \delta_{\mathrm{IAPA}}^{i} .
\end{aligned}
$$

Each $\Pi_{\mathrm{as}}^{i}$ consists of a fixed zeroth-order bound orbital and a Gamow orbital carrying the decay information for each channel. Within IAPA, the assumption is that interchannel coupling does not affect significantly the radial characteristics of each $\Pi_{\mathrm{as}}^{i}$. Thus, in the subsequent computation of interchannel coupling they are held fixed-a fact which makes the last step of our theory computationally economical. 
This step concerns interchannel coupling and involves the following: Having obtained each $\Pi_{\text {as }}^{i}$, we reconstruct the total non-Hermitian matrix with diagonal matrix elements $E_{0}$ and $\left\langle\Psi_{0}^{-2} \Pi_{\mathrm{as}}^{i}|H| \Psi_{0}^{-2} \Pi_{\mathrm{as}}^{i}\right\rangle$ for each channel $i$ and off-diagonal matrix elements $\left\langle\Psi_{0}|H| \Psi_{0}^{-2} \Pi_{\text {as }}^{i}\right\rangle$ and $\left\langle\Psi_{0}^{-2} \Pi_{\mathrm{as}}^{i}|H| \Psi_{0}^{-2} \Pi_{\mathrm{as}}^{i}\right\rangle$ for channels $i$ and $j$.

Due to the separate optimization of the $\Pi_{\mathrm{as}}^{i}$, nonorthonormality effects come up to the computation of the offdiagonal matrix elements. This implies the appearance of many new overlap, one- and two-electron integrals, which are computed explicitly. The diagonalized resonance function has the form:

$$
\Psi=\alpha \Psi_{0}+\sum_{i} c_{i}\left(\Psi_{0}^{-2} \otimes \Pi_{\mathrm{as}}^{i}\right)
$$

Then, each partial complex eigenvalue obtained from the mixing of the asymptotic pairs (MAP), $z_{\text {MAP, }}^{i}$ is given by

$$
\begin{aligned}
z_{\mathrm{MAP}}^{i}-E_{0} & =\frac{c_{i}}{a}\left\langle\Psi_{0}|H| \Psi_{0}^{-2} \Pi_{\mathrm{as}}^{i}\right\rangle \\
& \equiv \delta_{\mathrm{MAP}}^{i}-(i / 2) \gamma_{\mathrm{MAP}}^{i}
\end{aligned}
$$

The mixed total width and energy shift are then given by

$$
\begin{aligned}
& \Gamma_{\mathrm{MAP}}=\sum_{i} \gamma_{\mathrm{MAP}}^{i}, \\
& \Delta_{\mathrm{MAP}}=\sum_{i} \delta_{\mathrm{MAP}}^{i} .
\end{aligned}
$$

\section{APPLICATION TO THE Ne $1 s 2 s^{2} 2 p^{62} S$ AUTOIONIZING STATE}

The choice of this state was already made in Ref. 7. It is a nontrivial nine-electron system for which the standard complex rotation methods (see Refs. 5, 7, 13, 14, 16, 18, 23 , and references therein) have severe limitations.

Its energy has been measured to be $870.3 \mathrm{eV}$ above the ground state, ${ }^{35}$ in perfect agreement with theory. ${ }^{13}$

The information on the partial and total widths is given in Table I. There we show the experimental values ${ }^{36,37}$ and theoretical results from four types of many-electron calculations: Two with real coordinates which involve bound-continuum orbital mixing and interchannel coupling, ${ }^{37,38}$ and two from our work which involves the many-body theory in the complex energy-coordinate planes.

The $1 s 2 s^{2} 2 p^{62} S$ state has five asymptotic-pair correlation functions corresponding to the transitions $1 s-2 p^{21} D$, $1 s-2 p^{2}{ }^{1} S, \quad 1 s-2 s 2 p^{3} P^{o}, \quad 1 s-2 s 2 p^{1} P^{o}, 1 s-2 s^{2}{ }^{1} S$. These functions were computed in Ref. 13 within the IAPA and the approximation that, for $\mathrm{Ne}^{+}, \Psi_{0}$ is represented well by its Hartree-Fock representation. [No Fermi-sea or symmetric exchange of orbital symmetry (SEOS) correlations are present. ${ }^{25}$ ]

The effect of interchannel coupling within our scheme is not large. We note that $\Delta_{\text {MAP }}=-0.08 \mathrm{eV}$ while $\Delta_{\text {IAPA }}=-0.09 \mathrm{eV}$ (Ref. 13). Both for IAPA and MAP, the trends of the partial widths follow those of the experiment. ${ }^{36,37}$ For the strongest transitions $1 s-2 p^{2}{ }^{1} D$ and $1 s$ $2 s 2 p{ }^{1} P^{o}$, the agreement of MAP with experiment is very good. For the total widths the discrepancy is $15 \%$. However, the experimental value is given an error of $10 \% .{ }^{37}$

\section{CONCLUSION}

The present work on the partial and total widths of a many-electron, multichannel autoionizing state has completed a series of analyses and results on the quantum mechanics of resonances in terms of complex coordinates. $5,7,8,13,19$ It has been shown how the seemingly large and very complicated problem of obtaining reliable and verifiable information on arbitrary resonances can be reduced to a transparent and manageable one whose solution includes relaxation, localized and asymptotic correlation, and interchannel coupling effects.

We have chosen the $\mathrm{Ne}^{+} 1 s 2 s^{2} 2 p^{62} S$ Auger state as a good test case for our theory. In order to appreciate this better, we refer to the recent review by Ho (Ref. 16) where the difficulties for partial-width calculations are stated explicitly and where results for only two- and three-electron, single-channel systems are presented. Further under-

\begin{tabular}{|c|c|c|c|c|c|c|}
\hline \multirow[b]{3}{*}{ Transition } & \multicolumn{5}{|c|}{ Theory } & \multirow[b]{3}{*}{$\begin{array}{c}\text { Experiment } \\
\text { Refs. } 36 \text { and } 37\end{array}$} \\
\hline & \multicolumn{2}{|c|}{ Complex coordinates } & \multicolumn{3}{|c|}{$\begin{array}{c}\text { Real coordinates } \\
\text { Ref. } 37\end{array}$} & \\
\hline & $\begin{array}{c}\text { IAPA } \\
\text { (Ref. 13) }\end{array}$ & $\begin{array}{c}\text { MAP } \\
\text { (This work) }\end{array}$ & Ref. 38 & $\begin{array}{c}\text { Hartree-Fock } \\
\text { Basis set }\end{array}$ & $\begin{array}{c}\text { "Transition state" } \\
\text { Basis set }\end{array}$ & \\
\hline $1 s-2 p^{21} D$ & 0.560 & 0.565 & 0.493 & 0.606 & 0.550 & $0.604 \pm 0.06$ \\
\hline $1 s-2 p^{21} S$ & 0.048 & 0.040 & 0.077 & 0.095 & 0.083 & $0.089 \pm 0.009$ \\
\hline $1 s-2 s 2 p^{3} P^{o}$ & 0.029 & 0.032 & 0.049 & 0.101 & 0.070 & $0.063 \pm 0.006$ \\
\hline $1 s-2 s 2 p^{1} P^{o}$ & 0.154 & 0.165 & 0.137 & 0.195 & 0.150 & $0.174 \pm 0.017$ \\
\hline $1 s-2 s^{21} S$ & 0.044 & 0.043 & 0.049 & 0.060 & 0.045 & $0.060 \pm 0.006$ \\
\hline Total & 0.835 & 0.844 & 0.805 & 1.057 & 0.898 & $0.990 \pm 0.099$ \\
\hline
\end{tabular}
standing could come from examination of more systems and from critical appraisal of the choice and optimization of the function spaces for asymptotic correlation.

TABLE I. Auger widths (in $10^{-2}$ a.u.) for the Ne $1 s$ hole state. 
${ }^{1}$ T. Åberg and G. Howat, in Theory of the Auger Effect, Vol. 31 of Handbuch der Physik, edited by W. Mehlhorn (Springer, New York, 1982).

2C. A. Nicolaides, Phys. Rev. A 6, 2078 (1972); Nucl. Instrum. Methods 110, 231 (1973).

${ }^{3}$ C. A. Nicolaides and D. R. Beck, J. Chem. Phys. 66, 1982 (1977).

${ }^{4}$ C. A. Nicolaides and D. R. Beck, Phys. Lett. 60A, 92 (1977); 65A, 11 (1978).

${ }^{5}$ C. A. Nicolaides and D. R. Beck, Int. J. Quantum Chem. 14, 457 (1978).

${ }^{6}$ C. A. Nicolaides, Y. Komninos, and D. R. Beck, Phys. Rev. A 24, 1103 (1981).

${ }^{7}$ C. A. Nicolaides, Y. Komninos, and Th. Mercouris, Int. J. Chem. S15, 355 (1981).

${ }^{8}$ C. A. Nicolaides and E. Adamides, Phys. Rev. A 27, 1691 (1983).

${ }^{9}$ Y. Komninos, G. Aspromallis, and C. A. Nicolaides, Phys. Rev. A 27, 1865 (1983).

${ }^{10}$ C. A. Nicolaides, Y. Komninos, and D. R. Beck, Phys. Rev. A 27, 3044 (1983).

${ }^{11}$ C. A. Nicolaides, IEEE J. Quantum Electron. QE-19, 1781 (1983).

${ }^{12}$ G. Aspromallis, Y. Komninos, and C. A. Nicolaides, J. Phys. B 17, L151 (1984).

${ }^{13}$ C. A. Nicolaides, Th. Mercouris, and Y. Komninos, Int. J. Chem. 26, 1017 (1984).

${ }^{14}$ Th. Mercouris and C. A. Nicolaides, J. Phys. B 17, 4127 (1984).

${ }^{15}$ G. Aspromallis, C. A. Nicolaides, and Y. Komninos, J. Phys. B 18, L545 (1985).

${ }^{16} \mathrm{An}$ overview of the progress that had been made in the field of complex coordinates for atomic and molecular calculations until the spring of 1978, when the present theory was proposed (Ref. 5), can be found in the articles of Vol. 14 of the Int. J. Quantum Chem. (1978). A recent review by Y. K. Ho [Phys. Rep. 99, 1 (1983)] discusses certain applications to mainly two-electron systems and points out the unsuitability of the standard complex rotation method for the partial-width calculations, with or without interchannel coupling.

${ }^{17}$ R. Yaris and H. S. Taylor, Chem. Phys. Lett. 66, 505 (1979); T. Noro and H. S. Taylor, J. Phys. B 13, L377 (1980).
${ }^{18}$ C. N. McCurdy and T. N. Rescigno, Phys. Rev. A 20, 2346 (1979).

${ }^{19}$ Y. Komninos and C. A. Nicolaides, Chem. Phys. Lett. 78, 347 (1981).

${ }^{20}$ A. M. Dykhne and A. V. Chaplik, Zh. Eksp. Teor. Fiz. 40, 1427 (1961) [Sov. Phys.-JETP 13, 1002 (1961)].

21 J. N. Bardsley and B. R. Junker, J. Phys. B 5, L187 (1972).

${ }^{22}$ B. Simon, Ann. Math. 97, 247 (1973).

${ }^{23}$ B. R. Junker and C. L. Huang, Phys. Rev. A 18, 313 (1978); B. R. Junker, Int. J. Quantum Chem. 14, 371 (1978).

${ }^{24}$ This result (Ref. 5) is practically indispensible for rigorous approaches in many-electron systems. It has also been used in more recent publications [e.g., K. T. Chung and B. F. Davis, Phys. Rev. A 26, 3278 (1982)].

${ }^{25}$ D. R. Beck and C. A. Nicolaides, Phys. Rev. A 26, 857 (1982); Int. J. Quantum Chem. S14, 323 (1980).

${ }^{26}$ D. R. Beck and C. A. Nicolaides, Chem. Phys. Lett. 59, 525 (1978).

${ }^{27}$ The notions of a "stabilized root" is the heuristic tool for resonance studies from repeated diagonalization of large matrices. See Refs. 28-34.

${ }^{28}$ E. Holøien and J. Midtdal, J. Chem. Phys. 45, 2209 (1966); J. Phys. B 3, 592 (1970).

${ }^{29}$ L. Lipsky and A. Russek, Phys. Rev. 142, 59 (1966).

${ }^{30}$ H. S. Taylor, G. V. Nazaroff, and A. Golebiewski, J. Chem. Phys. 45, 2872 (1966).

${ }^{31}$ A. U. Hazi and H. S. Taylor, Phys. Rev. A 1, 1109 (1970).

${ }^{32}$ I. Eliezer and Y. K. Pan, Theor. Chim. Acta 16, 63 (1970).

${ }^{33}$ H. S. Taylor and A. V. Hazi, Phys. Rev. A 14, 2071 (1976).

${ }^{34} \mathrm{M}$. Bettendorff, R. J. Buenker, and S. D. Peyerimhoff, Mol. Phys. 50, 1363 (1983).

${ }^{35}$ T. D. Thomas and R. W. Shaw, Jr., J. Electron Spectrosc. Relat. Phenom. 8, 45 (1976).

${ }^{36}$ M. O. Krause, F. A. Stevie, L. J. Lewis, T. A. Carlson, and W. E. Moddeman, Phys. Lett. 31A, 81 (1970); U. Gelius, S. Svensson, H. Siegbahn, E. Basilier, A. Faxalv, and K. Siegbahn, Chem. Phys. Lett. 28, 1 (1974); S. Svensson, N. Martensson, E. Basilier, P. A. Malmqvist, U. Gelius, and K. Siegbahn, Phys. Scr. 14, 141 (1976).

${ }^{37}$ G. Howat, T. Aberg, and O. Goscinski, J. Phys. B 11, 1575 (1978).

${ }^{38}$ H. P. Kelly, Phys. Rev. A 11, 556 (1975). 